\title{
Anharmonicity of Cu-based shape-memory alloys in the vicinity of their martensitic transition
}

\author{
Alfons Gonzàlez-Comas, Lluís Mañosa, and Antoni Planes \\ Departament d'Estructura i Constituents de la Matèria, Universitat de Barcelona, Avinguda Diagonal 647, Facultat de Física, \\ E-08028 Barcelona, Catalonia, Spain \\ Michel Morin \\ Groupe d'Etudes de Métallurgie Physique et Physique des Matériaux, INSA, 20 Avenue Albert Einstein, 69621 Villeurbanne, France
}

(Received 6 July 1998)

\begin{abstract}
We present measurements of the complete set of third-order elastic constants (TOEC) of Cu-Al-Ni and $\mathrm{Cu}-\mathrm{Al}$-Be shape-memory alloys, as functions of temperature. These data quantify the elastic anharmonic behavior of these alloys on approaching their martensitic transformation. We provide experimental evidence that the martensitic transition in $\mathrm{Cu}$-based shape-memory alloys occurs at a fixed value $(\simeq 60 \mathrm{GPa})$ of the TOEC combination $C_{3}=(1 / 8 \sqrt{3})\left(3 C_{112}-2 C_{123}-C_{111}\right)$, which is the relevant combination for the shear mechanisms leading the bcc structure to the close-packed martensite. By means of a Landau model for elastic phase transitions, the value of $C_{3}$ has been estimated from the crystal structure of the martensitic phase. The value obtained conforms reasonably with that found experimentally. [S0163-1829(99)04001-1]
\end{abstract}

\section{INTRODUCTION}

The anharmonicity in solid crystals due to the nonlinearity of interatomic forces with respect to atomic displacements, is the origin of many important thermodynamic properties of crystals such as the thermal expansion or the deviation of the lattice specific heat at high temperatures with respect to Debye contribution. Moreover, the anharmonic nature of lattice vibrations in crystals plays an essential role in determining the lattice dynamics in the vicinity of structural phase transitions.

The martensitic transformation (MT) is a first-order diffusionless solid-solid phase transition exhibited by many bcc solids on lowering the temperature and/or by application of a stress. ${ }^{1}$ Owing to the technologically important shapememory properties exhibited by the Cu-based alloys, this transition has been widely studied on these materials and, particularly, a big effort has been devoted to the study of anomalies preceding the transformation in the hightemperature bcc phase ( $\beta$ phase). ${ }^{2}$ Especially remarkable is the temperature softening of the acoustic-phonon modes associated with the shear elastic constant $C^{\prime}\left[=\left(C_{11}-C_{12}\right) / 2\right]$ observed in Cu-based shape-memory alloys ${ }^{3,4}$ on approaching the transition temperature $T_{M}$. A simple description of the transformation mechanism from bcc to martensite is based on homogeneous lattice shear strains on the (110) plane in the [110] direction, and a shuffle given by the displacement of a phonon in the $\mathrm{TA}_{2}$ branch with a wavelength equal to the periodicity of the modulation of the lowtemperature phase. As the lattice distortion itself is an anharmonic phenomenon, an anharmonicity's enhancement on approaching the MT is expected.

This work is concerned with quantifying the anharmonicity in Cu-based shape-memory materials. Analysis of different alloy families has shown that the elastic anisotropy $A$ $\left(=C_{44} / C^{\prime}\right)$ reaches a constant value at the martensitic transformation temperature $T_{M} \cdot{ }^{4}$ This fact means that there is a coupling between elastic modes. Since such a mode coupling is a typical consequence of acoustic anharmonicity, a fixed anisotropy value suggests a common anharmonic character at the transition point for all $\mathrm{Cu}$-based alloys.

On the other hand, recent lattice-dynamics calculations in bcc transition metals ${ }^{5}$ have shown that the anharmonic phonon-phonon coupling is very strong. Particularly, the low-energy phonon modes connected to the structural phase transition from the bcc to the close-packed phase ${ }^{6}$ have been found to exhibit the strongest anharmonic effects. In $\mathrm{Cu}-$ based alloys, the whole $\mathrm{TA}_{2}$ branch exhibits anomalous temperature dependence, ${ }^{7}$ and the vibrational anharmonicity can be well described by that of long-wavelength acoustic phonons, which is quantified by third-order elastic constants (hereafter abbreviated as TOEC).

Experimental data of the temperature dependence of TOEC's are particularly scarce for Cu-based alloys. Up to date, complete measurements are only available on $\mathrm{Cu}-\mathrm{Zn}-\mathrm{Al},{ }^{8}$ although room-temperature sets have been reported for $\mathrm{Cu}-\mathrm{Au}-\mathrm{Zn},{ }^{9} \mathrm{Cu}-\mathrm{Al}-\mathrm{Pd},{ }^{10}$ and $\mathrm{Cu}-\mathrm{Al}-\mathrm{Ni} .{ }^{11}$

Here we present measurements of the temperature dependence of TOEC of $\mathrm{Cu}-\mathrm{Al}-\mathrm{Ni}$ and $\mathrm{Cu}-\mathrm{Al}-\mathrm{Be}$ shape-memory alloys. They have been obtained by measuring the changes in ultrasonic wave velocities under applied uniaxial stress. In order to describe the vibrational behavior in the vicinity of the MT, measurements have been conducted on a suitable range of temperatures for each alloy. Results will be compared with the predictions of present Landau theories for MT, and will be discussed in terms of the stability of $\beta$ phase and nucleation mechanism of the martensite.

\section{EXPERIMENTAL DETAILS}

Two single crystals of the $\beta$ phase of $\mathrm{Cu}-\mathrm{Al}-\mathrm{Ni}$ (Cu: 68.1, Al: 28.1 and $\mathrm{Ni}: 3.8$ at\%) and $\mathrm{Cu}-\mathrm{Al}-\mathrm{Be}(\mathrm{Cu}: 74.1, \mathrm{Al}: 23.1$ and Be: 2.8 at\%) alloys were investigated. Crystals of centimeter dimensions were grown by the modified Bridgman process. We have used a low speed diamond saw to cut cubic shaped specimens with faces parallel to the (110), (110), and 
TABLE I. Combinations of applied uniaxial stress with elastic modes. $\mathbf{M}, \mathbf{N}, \mathbf{U}$ are, respectively, the directions of applied stress, propagation and polarization vectors.

\begin{tabular}{cccccccccc}
\hline \hline No & 1 & 2 & 3 & 4 & 5 & 6 & 7 & 8 & 9 \\
Mode & $C_{L}$ & $C^{\prime}$ & $C_{44}$ & $C_{11}$ & $C_{44}$ & $C_{44}$ & $C_{L}$ & $C^{\prime}$ & $C_{44}$ \\
\hline $\mathbf{M}$ & {$[001]$} & {$[001]$} & {$[001]$} & {$[110]$} & {$[110]$} & {$[110]$} & {$[1 \overline{1} 0]$} & {$[110]$} & {$[1 \overline{1} 0]$} \\
$\mathbf{N}$ & {$[110]$} & {$[110]$} & {$[110]$} & {$[001]$} & {$[001]$} & {$[001]$} & {$[110]$} & {$[110]$} & {$[110]$} \\
$\mathbf{U}$ & {$[110]$} & {$[1 \overline{1} 0]$} & {$[001]$} & {$[001]$} & {$[110]$} & {$[1 \overline{1} 0]$} & {$[110]$} & {$[1 \overline{1} 0]$} & {$[001]$} \\
\hline \hline
\end{tabular}

(001) planes, with an accuracy better than $2^{\circ}$. The samples were polished flat to surface irregularities of about $2 \mu \mathrm{m}$ and parallel to better than $10^{-3} \mathrm{rad}$. The size of the $\mathrm{Cu}-\mathrm{Al}-\mathrm{Ni}$ specimen was $10.20 \times 10.05 \times 8.95 \mathrm{~mm}^{3}$, and the $\mathrm{Cu}-\mathrm{Al}-\mathrm{Be}$ one was $9.7 \times 9.6 \times 7.95 \mathrm{~mm}^{3}$. The $\mathrm{Cu}-\mathrm{Al}-\mathrm{Ni}$ crystal transforms martensitically towards a $2 \mathrm{H}$ (Ramsdell notation) structure at $T_{M}=220 \mathrm{~K}$, and $\mathrm{Cu}-\mathrm{Al}-\mathrm{Be}$ towards a $18 \mathrm{R}$ structure at $T_{M}=261 \mathrm{~K}$. Densities at room temperature, 7096 $\mathrm{kg} \mathrm{m}^{-3}$ for $\mathrm{Cu}-\mathrm{Al}-\mathrm{Ni}$ and $7478 \mathrm{~kg} \mathrm{~m}^{-3}$ for $\mathrm{Cu}-\mathrm{Al}-\mathrm{Be}$, were computed from the measured lattice parameters. ${ }^{12}$

Quartz transducers driven at their fundamental frequency of $10 \mathrm{MHz}$ (X cut for longitudinal, $\mathrm{Y}$ cut for shear waves) were acoustically coupled to the surface of the sample by using Dow Resin 276-V9. Ultrasonic-pulse transit times were obtained using the phase-sensitive detection technique. To avoid the problem of calculating the stress-induced changes in crystal dimensions, the "natural velocity $W$ ", technique was used. ${ }^{13}$

Uniaxial stresses up to $10^{7} \mathrm{~Pa}$ were applied with an universal tensile machine equipped with compression grips and a cryofurnace which enables conducting measurements in the temperature range $240-340 \mathrm{~K}$. A reinforced stainless-steel plate was mounted at the bottom of the upper compression grip, with a spherical stainless-steel ball between the plate and the surface of the grip; this design minimized any possible shear component of the applied stress, thus ensuring that once the specimen was sandwiched between this plate and the lower grip, it was subjected to a truly uniaxial stress.

During compressive runs it was essential to ensure that velocity measurements were made at the same controlled temperature within $\pm 0.3{ }^{\circ} \mathrm{C}$ because ultrasonic wave velocity strongly depends upon temperature. Furthermore, temperature was continuously monitored with a thermocouple attached to the specimen in order to correct data from possible temperature drifts.

\section{EXPERIMENTAL RESULTS}

For a cubic crystal belonging to the crystallographic point group $m 3 m$, there are six independent third-order elastic stiffness tensor components $C_{i j k}$ (Voigt notation). They can be obtained from the initial slope of the uniaxial stress dependence of the ultrasonic wave velocities. The effect of uniaxial compression on the natural velocities of the mode configurations listed in Table I has been measured in $\mathrm{Cu}-$ $\mathrm{Al}-\mathrm{Ni}$ and $\mathrm{Cu}-\mathrm{Al}-\mathrm{Be}$ crystals at different temperatures. Figure 1 shows an example of velocity changes measured in $\mathrm{Cu}-\mathrm{Al}-\mathrm{Be}$ due to uniaxial stress at room temperature; curve numbers (1)-(9) correspond to those given in Table I. For each curve, data points were taken during both increasing (filled symbols) and decreasing (open symbols) compressive runs. The room-temperature data for $\mathrm{Cu}-\mathrm{Al}-\mathrm{Ni}$ were given in a preliminary report. ${ }^{11}$ For the two alloys investigated, velocity changes increase linearly with compression in the stress range measured. Hence, we can compute the stress derivative of the relative change in natural velocity, evaluated at zero stress $\{d(\Delta v / v) / d \sigma)\}_{\sigma=0}$, for each mode combination by means of a linear fit to the data. Figure 2 compiles the obtained stress derivatives at different temperatures for mode combinations given in Table I. It is apparent that the slow shear modes (labeled 2 and 8) associated with the elastic constant $C^{\prime}$ exhibit the larger sensitivity and temperature dependence to the applied stress. By replacing the values of $\{d(\Delta v / v) / d \sigma)\}_{\sigma=0}$ into the equations developed by Thurston and Brugger ${ }^{13}$ we computed numerically, using a leastmean-square method, the TOEC as functions of temperature. In $\mathrm{Cu}-\mathrm{Al}-\mathrm{Be}$, data previously obtained from hydrostatic pressure measurements ${ }^{14}$ have been added to the uniaxial data. This ensures a high reliability of the $C_{i j k}$ values computed for this alloy. The values obtained are shown in Fig. 3 for both alloys. The accuracy is estimated to be better than $8 \%$.

Although the use of the ultrasonic technique while applying a constant stress produces mixed TOEC $C_{i j k}^{S, T}$, the analysis of the relations between adiabatic and mixed TOEC (Ref. 15) does not lead to any significant correction for the two alloys. Consequently, data shown in Fig. 3 can be assumed to be purely adiabatic constants $C_{i j k}^{S}$ (this has been written without superscript $S$ throughout this paper). It is remarkable

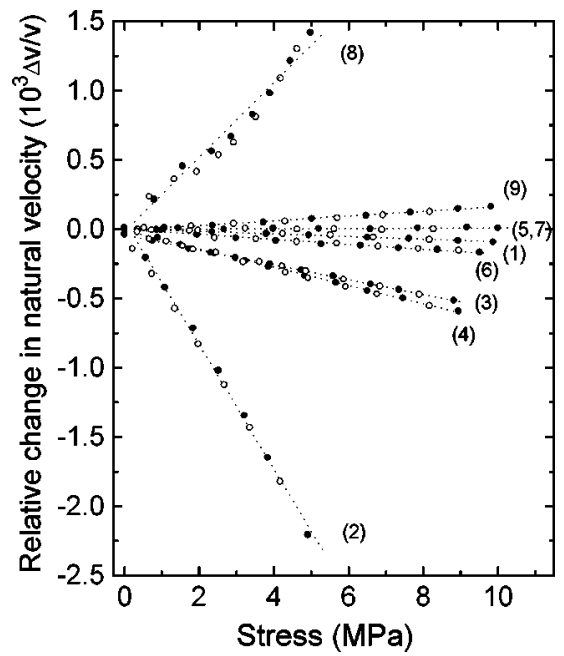

FIG. 1. Relative change in natural velocity of ultrasonic waves propagated in $\mathrm{Cu}-\mathrm{Al}-\mathrm{Be}$ alloy, induced by application of uniaxial stress at room temperature. Full and empty symbols correspond to increase and decrease compressive runs, respectively. Dashed lines are linear fits to the data. Curves have been labeled according to Table I. 

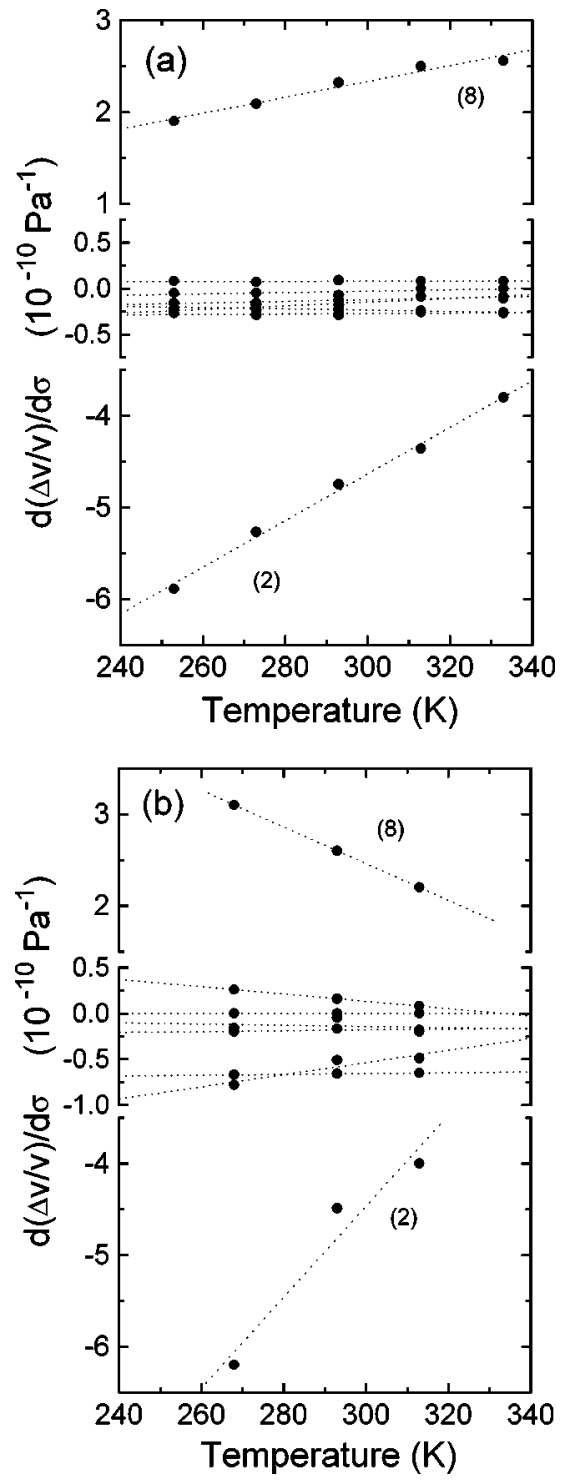

FIG. 2. Stress derivative of the relative change in natural velocity evaluated at zero stress, measured at different temperatures for $\mathrm{Cu}-\mathrm{Al}-\mathrm{Ni}$ (a) and $\mathrm{Cu}-\mathrm{Al}-\mathrm{Be}$ (b). For clarity only the labels for those modes exhibiting larger temperature change are indicated in the figure.

that all $C_{i j k}$ of both alloys are largely negative. The general trend is a decrease with increasing temperature. These characteristics are common for $\mathrm{Cu}$-based alloys and confirm that the premartensitic state of this kind of alloys is highly anharmonic. From atomic binding arguments Li et al. ${ }^{16}$ obtained for cubic symmetry the following relationships for TOEC: $C_{i j k}<0, C_{111}<C_{112}$ and $C_{112}<C_{123}$. Data for Cu-based alloys conform with these predictions.

\section{DISCUSSION}

The SOEC and TOEC are, respectively, the coefficients of the quadratic and cubic terms in the Landau elastic freeenergy expansion, and their values play an important role in determining several characteristics of an elastic phase transition such as the MT. Particularly, it has been shown ${ }^{17}$ that some combinations of TOEC (cubic invariants) are extremely useful in order to assess the nature of the transition.
The elastic free-energy expansion of a cubic crystal in terms of the symmetry adapted strain tensor components is given in Ref. 18. By considering only symmetry breaking terms, the expression adequate in our case is

$$
\begin{aligned}
\mathcal{F}= & \mathcal{F}_{\mathcal{O}}+\frac{1}{2} C^{\prime}\left(\eta_{1}^{2}+\eta_{2}^{2}\right)-\frac{1}{3} C_{3} \eta_{1}\left(\eta_{1}^{2}-3 \eta_{2}^{2}\right) \\
& +\frac{1}{4} C_{4}\left(\eta_{1}^{2}+\eta_{2}^{2}\right)^{2}
\end{aligned}
$$

where $C_{3}=(1 / 8 \sqrt{3})\left(3 C_{112}-2 C_{123}-C_{111}\right), C_{4}$ is a combination of fourth-order elastic constants, ${ }^{18}$ and $\eta_{1}$ $=(1 / \sqrt{3})\left(2 \eta_{33}-\eta_{22}-\eta_{11}\right)$ and $\eta_{2}=\eta_{22}-\eta_{33}$ are the shear strains associated with the shear mode $C^{\prime}$, expressed in terms of the Lagrangian strain tensor components $\left(\eta_{i j}\right)$. A linear combination of these two order parameters $\left(\eta_{1}, \eta_{2}\right)$ represents the symmetry-breaking displacement which yields the cubic structure towards the martensitic one (the modulation of this latter structure has not been considered here). The value of the third-order cubic invariant $C_{3}$ vanishes in a system undergoing a second-order transition, while nonzero values correspond to a first-order one. We have computed this relevant TOEC combination on a wide range of temperatures in $\mathrm{Cu}-\mathrm{Al}-\mathrm{Be}$ and $\mathrm{Cu}-\mathrm{Al}-\mathrm{Ni}$ from our measured TOEC data. Results are plotted in Fig. 4 as a function of the reduced temperature $T-T_{M}$. We have also included data for $\mathrm{Cu}$ $\mathrm{Zn}$-Al extracted from Ref. 8. In all alloys considered $C_{3}$ shows an unambiguous increase as the temperature is decreased towards the transition. At this point, $C_{3}$ has a nonvanishing value, in agreement with the first-order character of the MT. A marked enhancement of the anharmonicity of the (110)[1시 shear mode in the vicinity of the phase transition has already been qualitatively signaled, both for bcc (Refs. 9, 10) and for the martensitic phase, ${ }^{19}$ as a significant phenomenon exhibited by all $\mathrm{Cu}$-based alloys. However, up to the date, any attempt has been done in order to achieve the daunting task of evaluating quantitatively this anharmonicity over a wide temperature range in a family of alloys.

Another important feature is observed when the value of the cubic invariant is estimated by extrapolation at the transition point $T_{M}$ using a suitable linear fit for the data of each alloy. Although each alloy has a different slope $(\mathrm{Cu}-\mathrm{Al}-\mathrm{Ni}$ : $-0.14 \mathrm{GPa} \mathrm{K}^{-1}$, Cu-Al-Be: $-0.09 \mathrm{GPa} \mathrm{K}^{-1}$, and $\mathrm{Cu}-\mathrm{Zn}$ Al: $-0.05 \mathrm{GPa} \mathrm{K}^{-1}$ ), extrapolation to $T_{M}$ renders the same value of $C_{3}=60 \pm 1 \mathrm{GPa}$ for all the investigated alloys. This result suggests some kind of common anharmonic behavior at the transition point for all $\mathrm{Cu}$-based alloys, analogous to what has been reported for the elastic anisotropy. ${ }^{4}$ Until now, to our knowledge, there is no theory predicting a constant value of $C_{3}$ at $T_{M}$. Notwithstanding, in different theoretical approaches to the MT, this TOEC combination has been recognized to be crucial in the mechanisms driving the system towards the transition. ${ }^{20}$

Using Eq. (1) the value of $C_{3}$ at the transition point can be related to the shear strains at the martensitic structure as

$$
C_{3}=C^{\prime} \frac{\eta_{1}}{\eta_{2}^{2}} .
$$

By using Eq. (2), Gooding and Krumhansl ${ }^{21}$ made an estimation of $C_{3}$ for a $\mathrm{Ni}_{x} \mathrm{Al}_{1-x}$ alloy transforming to a $7 \mathrm{R}$ 

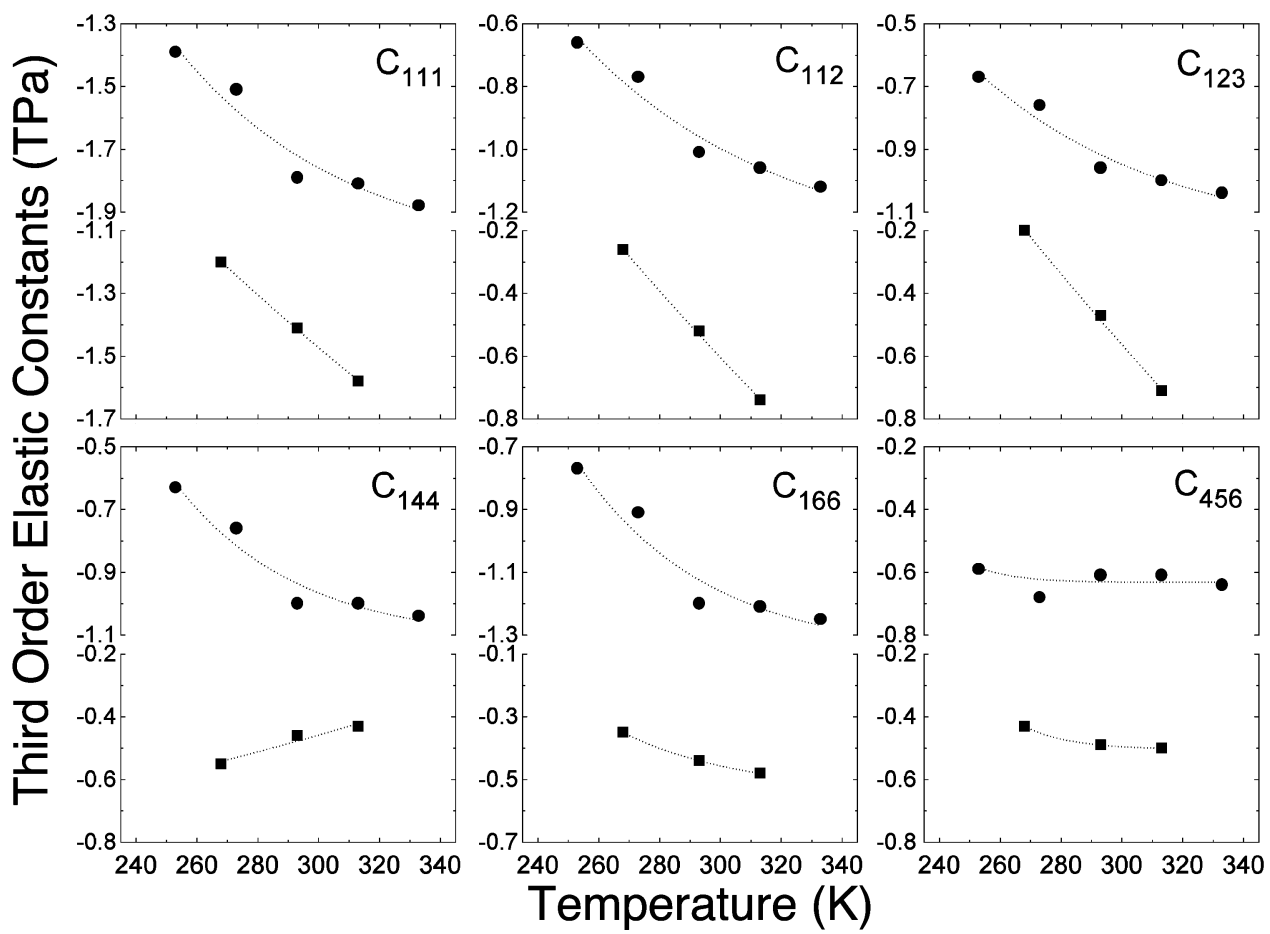

FIG. 3. TOEC of $\mathrm{Cu}-\mathrm{Al}-\mathrm{Ni}$ (circles) and $\mathrm{Cu}-\mathrm{Al}-\mathrm{Be}$ (squares) as functions of temperature. Their martensitic transition temperatures are 220 and $261 \mathrm{~K}$, respectively. Lines are guides to the eye.

structure. They obtained the values of $\eta_{1}$ and $\eta_{2}$ by relating these shear strains to the actual lattice distortion which was quantified by making use of the cell parameters of the $7 \mathrm{R}$ martensite of that alloy. As mentioned by these authors, the lack of experimental data for TOEC prevented them to check the validity of this theory. Now, we are in a position of evaluating this combination in the case of Cu-based alloys, and make a comparison with the measured value. As an example we use a $\mathrm{Cu}-\mathrm{Zn}-\mathrm{Al}$ alloy transforming to the $18 \mathrm{R}$ martensite structure.

Following the procedure described in Ref. 21, we have derived an expression of the lattice vectors of the $18 \mathrm{R}$ close-

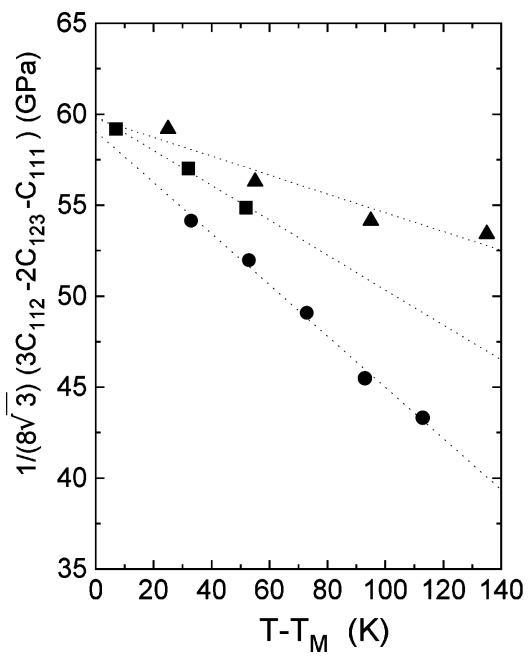

FIG. 4. TOEC combination $C_{3}$ as a function of the reduced temperature $T-T_{M}$. Cu-Al-Ni (circles) and $\mathrm{Cu}-\mathrm{Al}-\mathrm{Be}$ (squares) values have been computed from data shown in Fig. 3. Cu-Zn-Al data (triangles) have been computed from Ref. $8\left(T_{M}=158 \mathrm{~K}\right)$. Dashed lines are linear fits to the data. packed structure by applying the displacement pattern $\mathbf{u}(\mathbf{r})$ $=\eta_{2}(y \hat{\mathbf{y}}-z \hat{\mathbf{z}})+\sqrt{3}\left|\eta_{1}\right|(y \hat{\mathbf{y}}+z \hat{\mathbf{z}}-2 x \hat{\mathbf{x}})$, originated by the $\eta_{1}$ and $\eta_{2}$ shear strains, to the appropriate bcc lattice vectors. ${ }^{22}$ The lattice parameters for the $18 \mathrm{R}$ martensite structure are known for a composition close to one of the samples investigated here. ${ }^{23}$ From them and using the bcc lattice parameter $a=2.86 \AA$, we find that $\eta_{1}=0.025$ and $\eta_{2}=-0.044$ reproduce the lattice parameters and monoclinic angle of the $18 \mathrm{R}$ unit cell within $3 \%$ difference. Using these values and $C^{\prime}\left(T_{M}\right)=6.64 \mathrm{GPa},{ }^{8}$ we estimate $C_{3}=86 \mathrm{GPa}$ from Eq. (2). This value is slightly larger than the measured one; however, considering the approximations involved in the computation of $\eta_{1}$ and $\eta_{2}$, the agreement is rather satisfactory.

Another important point that the knowledge of TOEC enables us to discuss deals with the mechanical stability of the bcc lattice, which is strongly influenced by vibrational anharmonicity. As a starting point we consider the elastic free energy barrier separating the two minima corresponding to the bcc and martensitic phases. Such a value can be calculated from the strain-energy expansion Eq. (1) as

$$
\Delta \mathcal{F} \simeq \gamma \frac{C^{\prime 3}}{C_{3}^{2}},
$$

where $\gamma$ is a positive constant. Owing to the temperature softening of $C^{\prime}$ (Ref. 4) and the increase of $C_{3}$, Eq. (3) announces a significant decrease of the energy barrier on approaching $T_{M}$ in all $\mathrm{Cu}$-based alloys. This will favor the instability of the bcc lattice. A typical relative change of the energy barrier with temperature in these alloys is found to be about $-410^{-3} \mathrm{~K}^{-1}$. The aforementioned behavior is intimately related to the nucleation problem in MT. The classical theory for first-order phase transitions, based on thermally activated processes, has been proved to be inapplicable 
in MT. In the localized soft mode theory, Clapp ${ }^{24}$ suggested that the MT is triggered by a strain-induced instability in special regions of the parent lattice, located around suitable defects. This theory was further developed by Guénin and Gobin $^{25}$ and by Verlinden and Delaey. ${ }^{26}$ As the stored elastic energy induced by local strains reaches a certain value, instability results. Such strain amplitudes are easily encountered in the vicinity of specific lattice defects like dislocations in the $\beta$ phase. Hence, nucleation is postulated as a fluctuationless mechanism taking place in those zones where the reduced critical nucleus size approaches the size of the unstable zone. The size of the unstable zone has been estimated, for instance, in the case of a dislocation with Burgers vector $\vec{b}=a[01 \overline{1}]$ ( $a$ is the lattice parameter). ${ }^{26}$ An unstable region with elliptical shape and characteristic lengths $l$ and $l / \sqrt{A}$, where $l \sim\left[a\left(C_{3} / \Delta \mathcal{F}\right)\right]^{1 / 3}$ and $A$ is the elastic anisotropy, is predicted. The results presented here show that the increase of $C_{3}$ and the decrease of $\Delta \mathcal{F}$ on approaching $T_{M}$ result in an increase of the unstable region near the transition point, thus favoring the nucleation of the martensitic phase.

Finally, it is worth mentioning that a fluctuationless mechanism for MT has also been proposed for the martensitic transformation in $\mathrm{Zr}$ and Ti alloys. ${ }^{27}$ Although in that case the lattice distortion is not given by a long-wavelength acoustic phonon, the theory proposed by Vul and Harmon ${ }^{27}$ has a number of similarities with the localized soft-mode theory. In order for the transition to occur, the anharmonic parameter of the cubic term in their free-energy expansion had to be positive and increase with decreasing temperature. Notice that this behavior parallels that found here for the third-order invariant $C_{3}$. They also predicted that, due to anharmonic effects, the amplitude of static oscillations around some specific defects increased with temperature decreasing. Again, this is also similar to the behavior found here for $\mathrm{Cu}$-based shape-memory alloys.

\section{SUMMARY AND CONCLUSIONS}

We have presented measurements of the temperature dependence of the complete set of TOEC for $\mathrm{Cu}-\mathrm{Al}-\mathrm{Ni}$ and $\mathrm{Cu}-\mathrm{Al}-\mathrm{Be}$ alloys. The third-order cubic invariant $C_{3}$ $=(1 / 8 \sqrt{3})\left(3 C_{112}-2 C_{123}-C_{111}\right)$ associated with the homogeneous (110)[110] shear strains takes a fixed value $(\simeq 60$ $\mathrm{GPa}$ ) at the transition temperature. This value has been found to be independent of the type of alloy and also of the specific structure of the martensitic phase. Results show that $C_{3}$ increases as the alloy approaches the MT, reflecting an increase in the anharmonicity of the long-wavelength acoustic modes which drive the bcc structure towards the lowtemperature phase. By means of a phenomenological theory, we have shown that such an increase of anharmonicity yields to a decrease of the energy barrier between the two crystallographic phases, and thus favors nucleation of the martensite. The results presented here corroborate the fluctuationless mechanism proposed for the martensitic transformation: thermal fluctuations are irrelevant and the effect of temperature is to change the elastic coefficients in such a way that the instability of the lattice surrounding defects increases.

\section{ACKNOWLEDGMENTS}

A.G. acknowledges financial support from DGICyT. This work has received financial support from the CICyT (Spain) Project No. MAT98-0315. The authors would like to thank Luc Delaey and R.J. Gooding for valuable discussions. The $\mathrm{Cu}-\mathrm{Al}-\mathrm{Ni}$ crystal was kindly provided by J. Zarestky and C. Stassis.
${ }^{1}$ L. Delaey, Materials Science and Technology, Vol. 5 Phase Transformations in Materials, edited by P. Haasen (VCH, Weinheim, 1991), p. 399.

${ }^{2}$ J. A. Krumhansl and Y. Yamada, Mater. Sci. Eng., A 127, 167 (1990)

${ }^{3}$ A. Planes, Ll. Mañosa, D. Ríos-Jara, and J. Ortín, Phys. Rev. B 45, 7633 (1992).

${ }^{4}$ A. Planes, Ll. Mañosa, and E. Vives, Phys. Rev. B 53, 3039 (1996).

${ }^{5}$ T. May, W. Müller, and D. Strauch, Phys. Rev. B 57, 5758 (1998)

${ }^{6} \mathrm{The}_{\mathrm{TA}}$ branch usually exhibits a marked dip at the wave numbers corresponding to these soft phonons.

${ }^{7}$ Ll. Mañosa, J. Zarestky, T. Lograsso, D. W. Delaney, and C. Stassis, Phys. Rev. B 48, 15708 (1993).

${ }^{8}$ B. Verlinden, T. Suzuki, L. Delaey, and G. Guénin, Scr. Metall. 18, 975 (1984).

${ }^{9}$ A. Nagasawa and A. Yoshida, Mater. Trans., JIM 30, 309 (1989).

${ }^{10}$ A. Nagasawa, A. Kuwabara, Y. Morii, F. Fuchizaki, and S. Funahashi, Mater. Trans., JIM 33, 203 (1992).

${ }^{11}$ A. Gonzàlez-Comas and Ll. Mañosa, Phys. Rev. B 54, 6007 (1996)

${ }^{12}$ Ll. Mañosa, J. Zarestky, and C. Stassis (unpublished).
${ }^{13}$ R. N. Thurston and K. Brugger, Phys. Rev. 133, A1604 (1964).

${ }^{14}$ M. A. Jurado, M. Cankurtaran, Ll. Mañosa, and G. A. Saunders, Phys. Rev. B 46, 14174 (1992).

${ }^{15}$ O. M. Krasil'nikov, Sov. Phys. Solid State 19, 764 (1977).

${ }^{16}$ D. Y. Li, X. F. Wu, and T. Ko, Philos. Mag. A 63, 585 (1991).

${ }^{17}$ J. K. Liakos and G. A. Saunders, Philos. Mag. A 46, 217 (1982).

${ }^{18}$ M. P. Brassington and G. A. Saunders, Phys. Rev. Lett. 48, 159 (1982).

${ }^{19}$ A. Gonzàlez-Comas, Ll. Mañosa, M. Cankurtaran, G. A. Saunders, and F. C. Lovey, J. Phys.: Condens. Matter 10, 9737 (1998).

${ }^{20}$ R. J. Gooding and J. A. Krumhansl, Phys. Rev. B 38, 1695 (1988).

${ }^{21}$ R. J. Gooding and J. A. Krumhansl, Phys. Rev. B 39, 1535 (1989).

${ }^{22}$ S. Kajiwara, Trans. Jpn. Inst. Met. 17, 435 (1976).

${ }^{23}$ P. L. Rodríguez, F. C. Lovey, G. Guénin, J. L. Pelegrina, M. Sade, and M. Morin, Acta Metall. Mater. 41, 3307 (1993).

${ }^{24}$ P. C. Clapp, Phys. Status Solidi B 57, 561 (1973).

${ }^{25}$ G. Guénin and P. F. Gobin, Metall. Trans. A 13A, 1127 (1982).

${ }^{26}$ B. Verlinden and L. Delaey, Metall. Trans. A 19A, 207 (1988).

${ }^{27}$ D. A. Vul and B. N. Harmon, Phys. Rev. B 48, 6880 (1993). 